Sonderabdruck aus „Der Züchter", 24. Band, Heft 9, 1954.

Springer-Verlag, Berlin.

(Aus dem Max-Planck-Institut für Züchtungsforschung, Abteilung für Kulturpflanzenzüchtung, Hamburg-Volksdorf.)

\title{
Untersuchungen über die Züchtung von Kartoffeln mit hohem Stärkeertrag.*
}

Von H. Börger, D. KöHLER und R. v. Sengbusch.

Mit 8 Abbildungen.

Der Erfolg der Kartoffelzüchtung hängt unter anderm von der Auswahl geeigneter Eltern und der Auffindung von Nachkommen mit bestimmten wertvollen Eigenschaften ab. Die Wahrscheinlichkeit für das Auftreten dieser wertvollen Genotypen steigt mit der Zahl der Sämlinge. Nun ist es aber für den Züchter unmöglich, alle Genotypen über Jahre hindurch zu vermehren, bis er ein sicheres Urteil über jeden Klon hat. Er nimmt daher eine Auslese vor, die den Zweck hat, möglichst frühzeitig (d.h. schon aus den Sämlingen oder den ersten Vermehrungsstufen) die wertlosen Genotypen zu entfernen, und zwar möglichst nur diese. Wie groß bei der Auslese auf jede einzelne Eigenschaft die Wahrscheinlichkeit ist, da $\beta$ die gewünschten Genotypen erhalten bleiben, das hängt ab:

r. Von der Modifikabilität der Eigenschaft unter den Bedingungen des Zuchtbetriebes.

2. Von der Zahl der Individuen jedes Genotypes. Je stärker eine Eigenschaft modifiziert wird, desto weniger wahrscheinlich entspricht die am Einzelindividuum gemessene Eigenschaft dem Genotyp, desto weniger ist also eine Sämlingsauslese am Platze. Mit steigender Zahl der Individuen eines Genotyps steigt aber auch die Wahrscheinlichkeit, da $B$ ihr durchschnittlicher Phänotyp dem Genotyp entspricht. Daher steigt die Sicherheit der Aussage über jede Eigenschaft mit zunehmender Individuenzahl einer Nachbaustufe, ebenso mit fortschreitender Vermehrung der Individuen vom A- zum B-Klon usw.

3. Von der Größe der Differenzen zwischen den vorhandenen Eigenschaftsanlagen. Je weiter eine Eigenschaft genetisch variiert, desto sicherer lassen sich die schlechten von den guten Genotypen trennen. Die Wirksamkeit der Auslese hängt also von dem Verhältnis der genetischen Variationsbreite zur modifikativen Streuung ab.

4. Von der Strenge der Auslese, d.h. von der Lage der Auslesegrenzen. Je strenger die Auslese ist, desto mehr erhöht sich zwar der relative Anteil der guten Genotypen gegenüber den schlechten, desto größer ist aber auch die Wahrscheinlichkeit, daß auch gute Genotypen und darunter evtl. die gesuchten verloren gehen.

* Orto Hahn zum 75. Geburtstag am 8. März I954.
Wir haben an Hand der in der Wulfsdorfer Stärkekartoffelzüchtung (BöRGER) gewonnenen Werte untersucht, wie die Auslese auf hohen Stärkegehalt und hohen Rohertrag (Produkt $=$ Stärkeertrag) unter Berücksichtigung der vorstehenden Gesichtspunkte am zweckmäßigsten vor sich gehen sollte. Da wir meinen, da $\beta$ diese Ergebnisse von allgemeinerem Interesse sind, teilen wir sie im folgenden mit.

\section{Material.}

Die Auslese auf hohen Stärkeertrag ging folgendermaßen vor sich: Die Knollen der nach Wuchs und Gesundheit positiv beurteilten Sämlingspflanzen wurden im Winter gewogen; gleichzeitig wurde ihr Stärkegehalt (mit der Stärkewaage für Einzelpflanzen nach HeIMERdinger und von SENGBUSCH) bestimmt. Hierbei wurden bereits in den ausgesprochenen Stärkekreuzungen die Sämlinge mit einem Stärkegehalt unter I $7 \%$ verworfen. Im folgenden Jahr wurden aus möglichst sämtlichen größeren Knollen der Sämlinge (durchschnittlich mehr als Io) A-Klone gezogen, die kranken Klone im Laufe der Vegetationsperiode entfernt und bei den gesunden im darauffolgenden Winter Stärkegehalt und Knollenertrag festgestellt. Auf Grund der Ergebnisse an den Sämlingen und ihren A-Klonen, die noch einen recht hohen Prozentsatz der Sämlinge ausmachten, erfolgte die entscheidende Auslese, d.h. eine sehr starke Einschränkung des Materials. Die verbleibenden, nun schon auf hohen Stärkeertrag ausgelesenen Klone wurden weiter geprüft.

\section{Ergebnisse.}

I. Auslese auf Stärkegehalt

a) Kreuzung Olympia $\times$ Aquila.

In Abb. I sind die Stärkeprozente der Sämlinge denen der A-Klone im Koordinatensystem gegenübergestellt. Zwischen beiden besteht eine Korrelation. Das bedeutet, daß die erste Voraussetzung für eine Auslese gegeben ist, da $B$ nämlich in der Geschwisterschaft ${ }^{1}$ erbliche Unterschiede enthalten sind, zweitens

\footnotetext{
1 Zur Unterscheidung von der "Nachkommenschaft“" bei generativ vermehrten Pflanzen sprechen wir bei vegetativ vermehrten Pflanzen von "Geschwisterschaften". Sämtliche Individuen aus der Kreuzung $A \times B$, wobei $\dot{A}$ und $B$ bestimmte Eltern repräsentieren sollen, sind ja Geschwister, unabhängig von ihrer Nachbaustufe.
} 
aber auch, daß schon der Sämling eine einigermaßen wahrscheinliche Aussage über seinen anlagemäßig vorhandenen Stärkegehalt erlaubt. Hier hätte also mit Erfolg ausgelesen werden können. Läge z. B. die Aus-

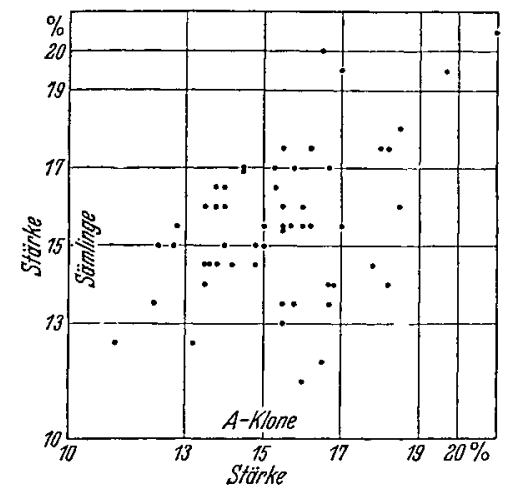

Abb. r. Vergleich der Stärkegehalte von Sämlingen und A-Klonen. Kreuzung Olympia $\times$ Aquila.

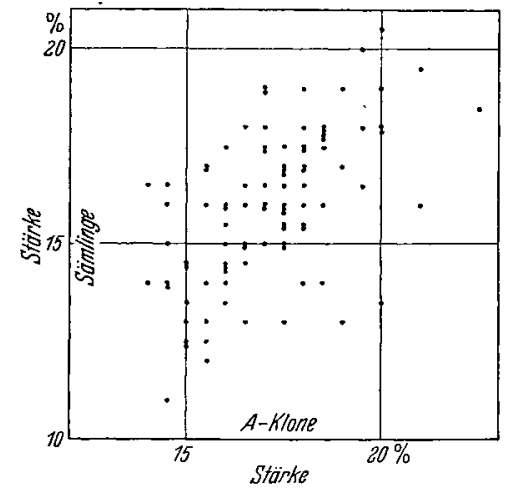

Abb. 2. Vergleich der Stärkegehalte von Sämlingen und A-Klonen. Kreuzung Ostbote $X$ Aquila.

lesegrenze bei den Sämlingen bei I3\% (bzw. I5\%, I $7 \%$, I $9 \%$ ), so wäre der durchschnittliche Stärkegehalt der übrigbleibenden 50 (bzw. 3I, 9, 4) A-Klone (von insgesamt 55 ) bei $15,6 \%$ (bzw. I6, I \%, I8, I \%, I8,8\%)

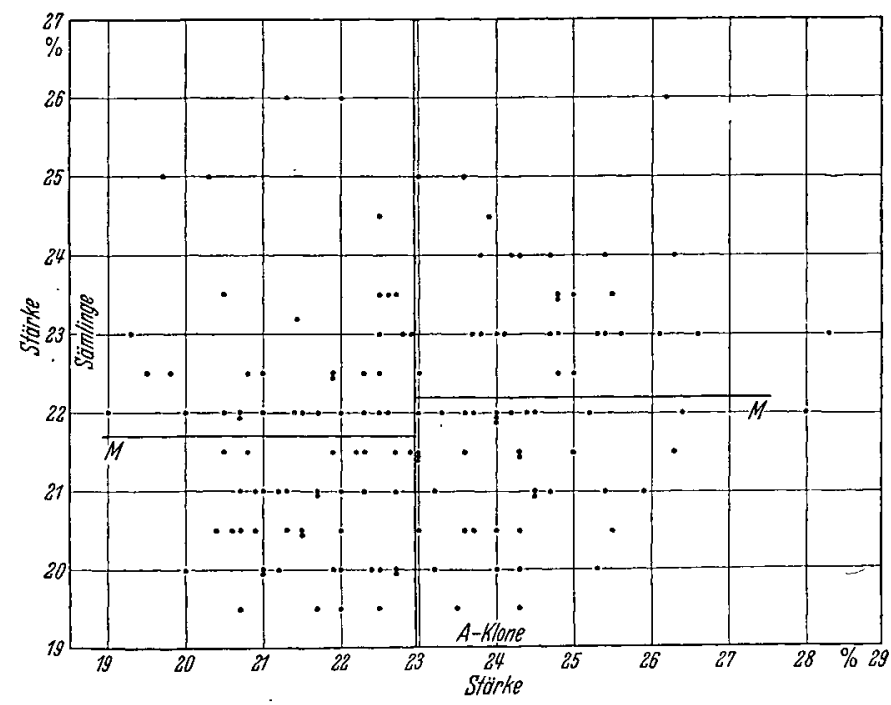

Abb. 3. Vergleich der Stärkegehalte von Sämlingen und A-Klonen. Kreuzung Falke $\times$ Hochprozentige

gewesen gegenüber dem Rest von 5 (bzw. 24, 46, 5I) A-Klonen mit einem durchschnittlichen Stärkegehalt von $\mathrm{I}_{4,7 \%}$ (bzw. I4,8\%, I5,0\%, I5,3\%). Die Auslese hätte also ihren $Z$ weck - Einschränkung des Materials auf Kosten der negativen Typen - erfüllt. Da in die- sem Beispiel die größte Differenz der durchschnittlichen Stärkegehalte der positiven Klone zwischen den Auslesestufen $I_{5} \%$ und $I 7 \%$ liegt, hätte die Auslesegrenze am zweckmäßigsten bei $17 \%$ angesetzt werden müssen. Das hätte zur Folge gehabt, daß nur etwa der fünfte Teil der A-Klone hätte aufgezogen werden müssen, oder da $B$ bei gleicher A-Klonzahl die Sämlingszahl fünfmal so hoch hätte sein können. Im letzteren Fall wären selbstverständlich die Aussichten, auch in anderer Beziehung erwünschte Klone zu finden, fünfmal so groß gewesen. - Einen entsprechenden Fall zeigt Abb. 2.

b) Kreuzung Falke $\times$ Hochprozentige.

I42 A-Klone wurden auf ihren Stärkegehalt untersucht. Der Vergleich ihres Stärkegehaltes mit dem der Sämlinge (Abb. 3) zeigt, da $\beta$ zwischen beiden nur eine ganz schwache Korrelation besteht. Dies könnte bedeuten, daß in der Geschwisterschaft überhaupt keine erblichen Unterschiede im Stärkegehalt auftreten, die Korrelation also nur vorgetäuscht ist, oder da B die modifikative Variabilität die genetische erheblich übertrifft. Eine Entscheidung zwischen beiden Möglichkeiten wird erst durch den Vergleich zwischen A- und B-Klonen möglich (Abb. 4). Auch hier zeigt sich wieder eine schwache Korrelation. Da diese auch in späteren Nachbaustufen erhalten bleibt, gibt es also erbliche Unterschiede. Trotzdem wäre innerhalb der Geschwisterschaft eine Auslese am Sämling oder AKlon (fast) ohne Erfolg geblieben und hätte nur zu einer Einschränkung des Materials geführt.

Bei gemeinsamer Auslese der unter a und $b$ beschriebenen Geschwisterschaften, wie sie ja normalerweise durchgeführt wird, hätte die Entfernung aller Sämlinge mit einem Stärkegehalt unter $\mathrm{I} 9 \% \mathrm{zu}$ fast vollständiger Trennung der beiden Geschwisterschaften geführt. Dies ist ein sehr anschauliches Beispiel dafür, daß die Auslese um so besser wirken kann, je mehr die genetische Variation die Modifikation einer Eigenschaft übertrifft. 
prozente aller Kreuzungen mit der Sorte „Hochprozentige" über allen anderen Kreuzungen. Die "Hochprozentige" vererbt hohen Stärkegehalt also dominant. $\mathrm{Ob}$ sie homozygot hochprozentig ist, ist nicht sicher, aber sehr wahrscheinlich. Die Zahl der wegen eines Stärkegehaltes unter I7\% entfernten
Nachbaustufen notwendig, um zu einem sicheren Urteil zu kommen.

\section{Stärkeertrag.}

Nach Abb. 7 könnte man der Meinung sein, daß zwischen Stärkegehalt und Ertrag eine negative Kor-

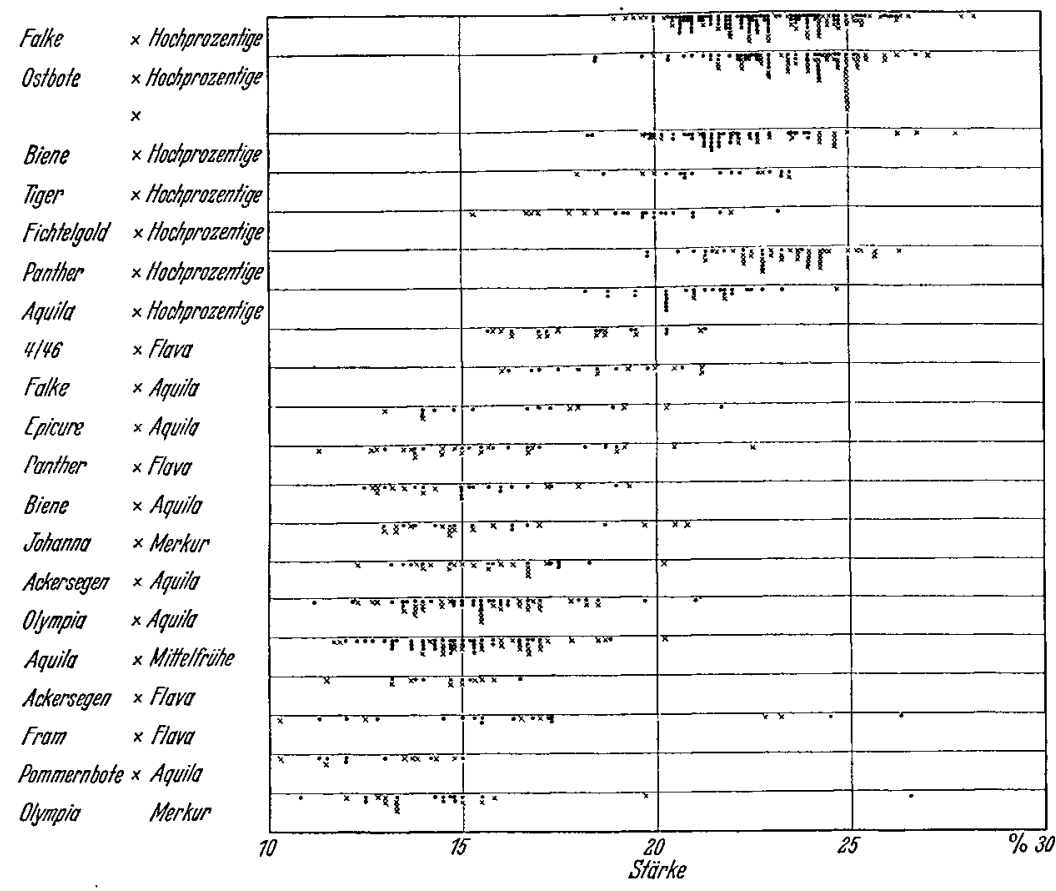

Abb. 5. Stärkegehalte der A-Klone aus verschiedenen Kreuzungen. Der Stärkegehalt des Sämlings weicht um weniger $(\cdot)$ oder mehr $(X)$ als $1 / 10$ von dem Stärkegehalt des A-Klons ab.

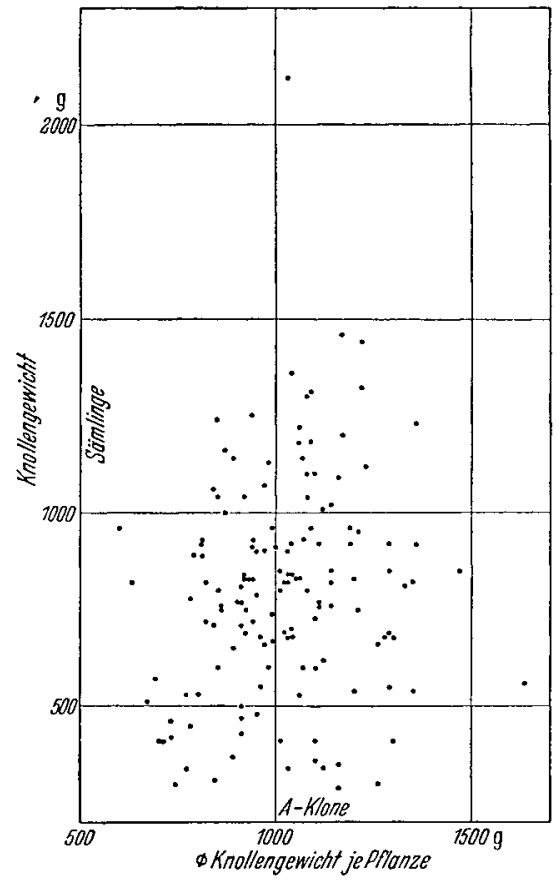

Abb. 6. Vergleich der Sämlings- und A-Klonerträgc. Kreuzung Falke $\times$ Hochprozentige.

Sämlinge ist nicht mehr festzustellen. In der Kreuzung „Fram" $\times$,,Flava“" treten einige sehr hochprozentige Typen auf. Falls es sich dabei nicht um eine Verwechslung oder Mutation handelt, könnten diese Typen nur durch Kombination recessiver Gene für Stärkereichtum entstanden sein. In diesem Fall könnte diese Kreuzung als Hinweis dafür dienen, daß es zur Auswahl der Kreuzungseltern nicht genügt, kleine Testkreuzungen durchzuführen und nur die Kreuzungen in größerem Umfang zu untersuchen, deren Nachkommen einen durchschnittlich hohen Stärkegehalt besitzen. Solche extremen Typen, wie sie in der Geschwisterschaft aus "Fram" $X$,Flava“ auftreten, würden dann niemals gefunden werden.

\section{Ertragsauslese.}

Es ist zu überprüfen, bei welcher Nachbaustufe die Ertragsauslese begonnen werden kann. Wenn überhaupt, dürfte es nur sehr wenige Geschwisterschaften geben, bei denen zwischen Sämlings- und A-Klonertrag eine deutliche Korrelation besteht. Abb. 6 zeigt als allgemein gültiges Beispiel die Kreuzung ,Falke“ $\times$,Hochprozentige“. Eine Sämlingsertragsauslese ist also meistens sinnlos. Dagegen bietet schon der Ertrag eines größeren A-Klons (siehe unter Material) einen gewissen Anhaltspunkt für den echten Ertrag des Klons, wie aus Abb. 7 hervorgeht. Trotzdem ist nach allem bisher darüber Bekanntem in Bezug auf Ertrag eine genaue Überprüfung der Klone der späteren

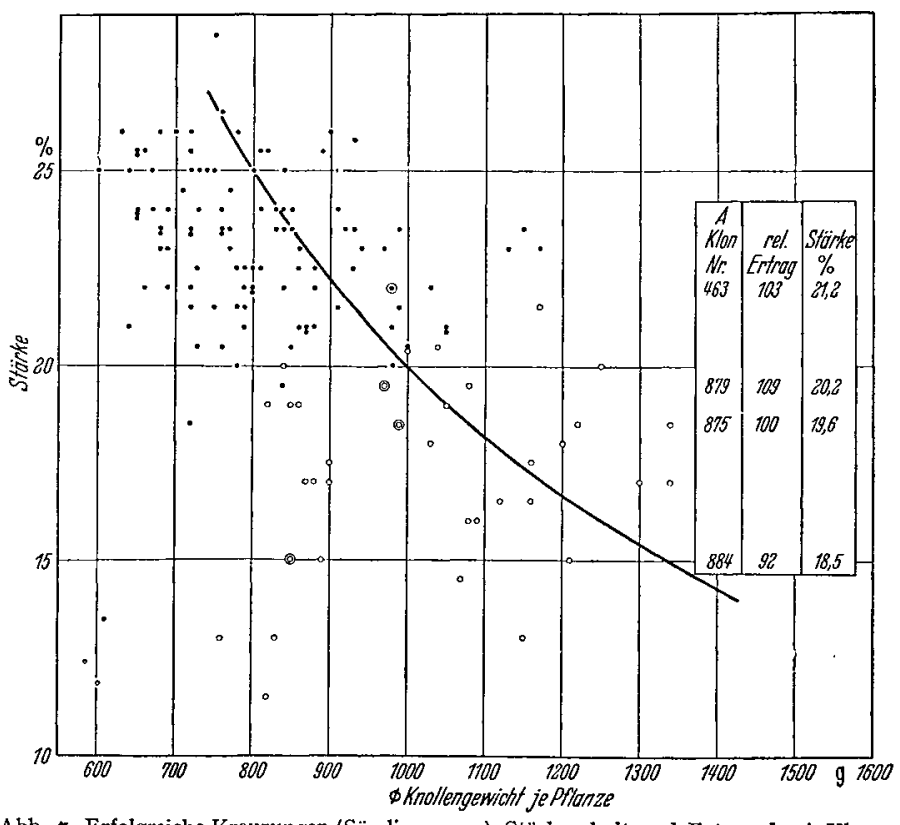

Abb. 7. Erfolgreiche Kreuzungen (Sämlinge 1949). Stärkegehalt und Ertrag der A-Klone. - Ostbote $\times$ Hochprozentige;

$38 / 46$ (demissum-Bastard) $\times$ Aquila;

○( Klone 1953 im Bundessortenamt geprüft; Werte der Prüfung rech ts angegeben.

- Die Linie verbindet die Werte, deren Produkt einem Stärkeertrag von $200 \mathrm{~g} /$ Pflanze entspricht.

relation besteht, d. h. also, daß man entweder nur Sorten mit hohem Stärkegehalt und mittlerem Ertrag oder mit mittlerem Stärkegehalt und hohem Ertrag züchten könnte. Diese Annahme dürfte wohl nicht berechtigt sein, wie aus Abb. 8 hervorgeht. Dort sind die Werte der A-Klone aus 4 verschiedenen Kreu- 
zungen eingetragen. Jede Geschwisterschaft verwirklicht durchschnittlich eine der 4 denkbaren Kombinationen zwischen hohem und mittlerem Ertrag und hohem und mittlerem Stärkegehalt. Hoher Ertrag und hoher Stärkegehalt sind also kombinierbar ${ }^{1}$.

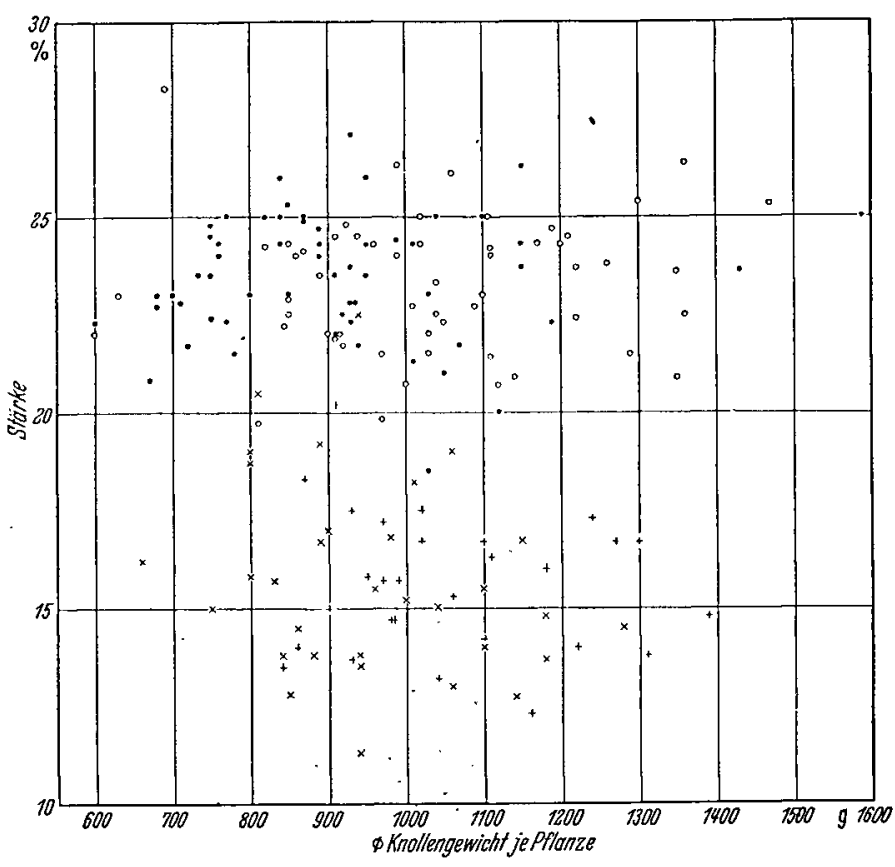

Abb. 8. Stärke und Ertrag der A-Klone von 4 Kreuzungen (Sämlinge I950).

$$
\begin{array}{ll}
- \text { Ostbote } & \times \text { Hochprozentige; } \\
\text { O Falke } & \times \text { Hochprozentige; } \\
+ \text { Ackersegen } & \times \text { Aquila; }
\end{array}
$$

So wenig berechtigt wie die Annahme, da $\beta$ die beiden Eigenschaften nicht $z u$ kombinieren wären, erscheint die, daß die Kombination der beiden Eigenschaften notwendig mit anderen Fehlern gekoppelt sei.

\section{Schlußbetrachtungen.}

Dem Züchter ist daran gelegen, seinen Genotypenbestand möglichst schnell auf die wertvollen Typen einzuengen. Dies gelingt ihm dadurch, daß eir einerseits in der Sämlings- oder in der frühesten Nachbau-

1 Neben dieser Kombinierbarkeit bleibt natürlich durchaus die Möglichkeit bestehen, da $\beta$ in bestimmten Geschwisterschaften eine negative Korrelation zwischen Ertrag und Stärkegehalt vorkommen kann. stufe, die ein einigermaßen sicheres Urteil über eine Eigenschaft zuläßt, mit der Auslese auf diese Eigenschaft hin beginnt, andererseits, daB er die Klone möglichst groß macht.

Durch die frühzeitige Einschränkung des Genotypenbestandes gewinnt er erstens die Möglichkeit, das neue Ausgangsmaterial zu vergrößern, also mit gröBeren Aussichten auf Auffindung bester Typen zu arbeiten, zweitens kann er dann seine Klone früh beurteilen und seine Sorten entsprechend früh auf den Markt bringen. Wir konnten die Vermutung; da $B$ schon Sämlinge erfolgreich auf Stärke ausgelesen werden können, bestätigen. Außerdem zeigte es sich, daß schon ein aus etwa Io Pflanzen bestehender A-Klon eine einigermaßen sichere Rohertrags- und damit Stärkeertragsfeststellung zuläßt.

So wird es verständlich, daß Klone der Wulfsdorfer Züchtung, die von Sämlingen des Jahres I949 abstammten, schon I953 im Versuch des Bundessortenamtes sehr positiv abschnitten. Wir konnten auBerdem nachweisen, daß hoher Stärkegehalt und hoher Rohertrag wenigstens in den bisher bekannten Größenordnungen kombinierbar sind.

Als besonders wertvolle Elternsorte erwies sich die stärkereiche ,Hochprozentige" (Züchter voN ZITZEwITZ). Diese Sorte ist in anderen Eigenschaften durchaus nicht überragend. Gerade darum aber bestätigt sie unsere Ansicht, daß es für den Züchter sehr wichtig sein kann, auch einseitig überragende Klone, die bei der Auslese nach anderen Eigenschaften verworfen werden würden, mit dem Ziel zu erhalten, diese positiven Eigenschaften später mit anderen gleichwertigen Eigenschaften zu kombinieren. Es-würde sich also lohnen, neben der normalen Auslese auch eine auf einzelne wesentliche Eigenschaften vorzunehmen. Man könnte z.B. nach noch stärkereicheren Elternsorten als "Hochprozentige" suchen (ev. sogar auf recessiver Basis, siehe Kreuzung „Fram“ $\times$,Flava“), deren Ertrag nicht hoch zu sein brauchte, und ebenso nach besonders ertragreichen Sorten, deren Stärkegehalt zunächst gleichgültig sein könnte, um schließlich die aufgefundenen überragenden Eigenschaften miteinander zu kombinieren. Auf diese Weise könnte man vielleicht in der. Züchtung stärkeertragreicher Kartoffeln noch einen Schritt weiter kommen.

Die in dieser Untersuchung gewonnenen Ergebnisse lassen sich sinngemä $ß$ auch auf andere vegetativ vermehrte Kulturpflanzen übertragen. 